\title{
Susceptibility of $S$. pneumoniae to Various Antibiotics Among Strains Isolated from Patients and Healthy Carriers in Different Regions of Brazil (1999-2000)
}

\author{
Rossi F., Andreazzi D., Maffucci M. and Pereira AA.
}

HC-FMUSP, Emilio Ribas Institute, São Paulo, SP; State University of Rio de Janeiro, RJ, Brazil

\begin{abstract}
Resistance of microbes to commonly used antibiotics became a major concern at the end of the last century. Because Streptococcus pneumoniae is the most common pathogen in respiratory infections, we conducted microbiological assessment of drug susceptibility patterns among strains collected from two different population groups: 1 ) adult and pediatric patients (375 isolates) with different infections, and 2) healthy children in day care centers ( $<5$ years old; 350 isolates). High level resistance to penicillin was not identified in either group. Intermediate resistance levels were similar in both groups (adults: 9.9\%; children: 9.2\%). The Central West region of Brazil tended to have lower susceptibility of S.pneumoniae from infected adults and children to penicillin (81\% vs. $93 \%$ in the South and $90 \%$ in the Southeast), tetracycline (64\% vs. 80\% and $76 \%)$, and trimethoprim/sulfamethoxazole (14\% vs. 34\%). Susceptibility was similar among strains from nasal cultures of healthy children tested in each of 4 regions of Brazil. All isolates were susceptible to cefaclor, cefotaxime and amoxacillin/clavulanate. This study, in two distinct populations, allowed characterization of local microbiological resistance patterns. This data is expected to be of use in guiding empiric therapy in the different regions of Brazil.

Key Words: S. pneumoniae, resistance, susceptibility, respiratory infection, antibiotics, Brazil.
\end{abstract}

There are many conditions favorable to the development of antimicrobial resistance in different countries throughout Latin America. Brazil is an important player in this situation since self-medication and over-the-counter sales of antibiotics are common practices still to be prevented.

Streptococcus pneumoniae is the most common bacterial pathogen associated with respiratory tract infections such as otitis media, sinusitis, and pneumonia in adults and children [1]. More than 1 million deaths per year in children less than 5 years of age are due to infections caused by Streptococcus pneumoniae [2]. Although efforts are being made to develop

Received on 14 March 2001; revised 10 December 2001.

Address for correspondence: Flávia Rossi, MD. Diretora do Laboratório de Microbiologia-HC-FMUSP. Rua Lee de Forest, 32. Zip Code: 04576-120. São Paulo-SP, Brazil. Fax: (55 11)37580457.E-mail: ffrossi@attglobal.net

The Brazilian Journal of Infectious Diseases 2001;5(6):305-312. (C) 2001 by The Brazilian Journal of Infectious Diseases and Contexto Publishing. All rights reserved.

$1413-8670$ effective vaccines, early diagnosis and care is the current approach to treat such infections. This includes a focus on standard microbiology procedures and a well designed quality assurance program, in order to ensure that results help to predict clinical outcome and also indicate adequate antimicrobial therapy. There are no Brazilian microbiological standards, so, most of our laboratories follow NCCLS (National Committee for Clinical Laboratory Standards, USA) guidelines. MIC (minimum inhibitory concentration) procedures are recommended to detect penicillin, amoxicillin, and cephalosporin resistance among Streptococcus pneumoniae since the disc diffusion method is not recommended [3]. Most of our clinical laboratories only use the disc diffusion method which may lead to a degree of uncertainty when determining drug resistance in such microorganisms.

The prevalence of Streptococcus pneumoniae resistance in Latin American countries is increasing, but current data show that Brazil's rate is relatively low compared to other countries worldwide [4]. 


\section{Objective}

This study was designed to determine susceptibility patterns of Streptococcus pneumoniae isolates to $\beta$ lactam and non- b-lactam agents from 1999 to 2000, in different geographical regions in Brazil (Figure 1). A surveillance program was designed in which two groups of isolates were obtained. Group A isolates were recovered from adult and pediatric patients presenting with various infections, and Group B isolates were obtained from healthy pediatric carriers (Table 1).

\section{Materials and Methods}

Two different groups were studied to determine susceptibility patterns among S. pneumoniae. Distinct Brazilian regions were enrolled in each group as shown in Table 1.

Group A. We collected 375 Streptococcus pneumoniae strains from four different Brazilian regions. Child (34\%) and adult (66\%) specimens were sent to microbiology laboratories for routine diagnostic cultures. Sputum samples were included only when the sample was considered significant according to Gram's stain scores and related to a confirmed clinical infection. Demographic information, other than age and infectious process related to the specimens, was not reported; this information was difficult to obtain from many patients.

Group B. We collected 2,521 nasal swabs from healthy children younger than 5 years old in daycare centers reporting no episodes of infection or antibiotic use in the previous month. Swabs were collected by the same nurse from the different Brazilian regions, and were sent to a central microbiology laboratory in São Paulo for culture, identificiation, and standard susceptibility testing.

$\underline{\text { Methods }}$

Identification was performed as recommended by the Manual of Clinical Microbiology ASM [5]. Isolates from both groups were tested against a panel of antibiotics using a combined method of disc diffusion and MIC by E-tests (AB Biodisc, Solona, Sweden). Susceptibility tests were performed with MuellerHinton agar supplemented with $5 \%$ sheep blood. Etests for penicillin, cefaclor, amoxicillin/clavulanate and cefotaxime were used according to the manufacturer's recommendations. Disc testing (Oxoid) for trimethoprim/sulfamethoxazole (TMP-SMZ), erythromycin, chloramphenicol, and tetracycline was performed following National Committee for Clinical Laboratory Standards (NCCLS M7-A5 / M2-A7 / M100 S10) procedures [3]. A single $150 \mathrm{~cm}$ agar plate with E-test strips and the discs were incubated for 20 to 24 hours in $5 \% \mathrm{CO}_{2}$. Testing of isolates was performed with concurrent testing of quality control organisms Streptococcus pneumoniae ATCC 49619 and Escherichia coli ATCC 35218. The range for susceptibility by E-test (susceptible, intermediate and resistant) are shown in Table 2. Pharmacokinetic/ pharmacodynamic (PK/PD) breakpoints were used for oral agents as recommended by the NCCLS M7-A5 / M2-A7 / M100 S10 [3] document.

\section{Results}

Group A: Patients. We obtained 375 Streptococcus pneumoniae isolates from three different Brazilian regions South (147); Southeast (186) and Center-West (42) from adults (248-66\%) and children (127-34\%). The isolates were obtained from respiratory tract infections in 136 (52\%), among which 98 (72\%) were from upper respiratory tract and 38 (28\%) were from lower respiratory tract infections. Other main sources of the isolates were blood in 120 patients $(32 \%)$ and CSF in 60 patients $(16 \%)$.

All of the isolates tested had MIC values to penicillin $<2.0 \mu \mathrm{g} / \mathrm{ml}$, no high level resistance according to NCCLS standards [3]. The rate of intermediate resistance to penicillin was $9.8 \%$ ( 37 of 375 isolates) The most active oral antimicrobial agent was amoxicillin/ clavulanate (100\% susceptible). Susceptibilities to other drugs were as follows: cefotaxime $100 \%$; cefaclor 
Table 1. Streptococcus pneumoniae: Group A(adult / pediatric infeccions) and Group B (pediatric carriers)

\begin{tabular}{llccc}
\hline Region & & Group A & \multicolumn{2}{c}{ Group B } \\
\hline North & $(\mathrm{N})$ & --- & 69 & $(373)$ \\
Northeast & $(\mathrm{NE})$ & --- & 51 & $(305)$ \\
Centerwest & $(\mathrm{CW})$ & 42 & --- & \\
Southeast & $(\mathrm{SE})$ & 186 & $130(1301)$ \\
South & $(\mathrm{S})$ & 147 & $100(542)$ \\
Total & & $\mathbf{3 7 5}$ & $\mathbf{3 5 0}(2521)$ \\
\hline
\end{tabular}

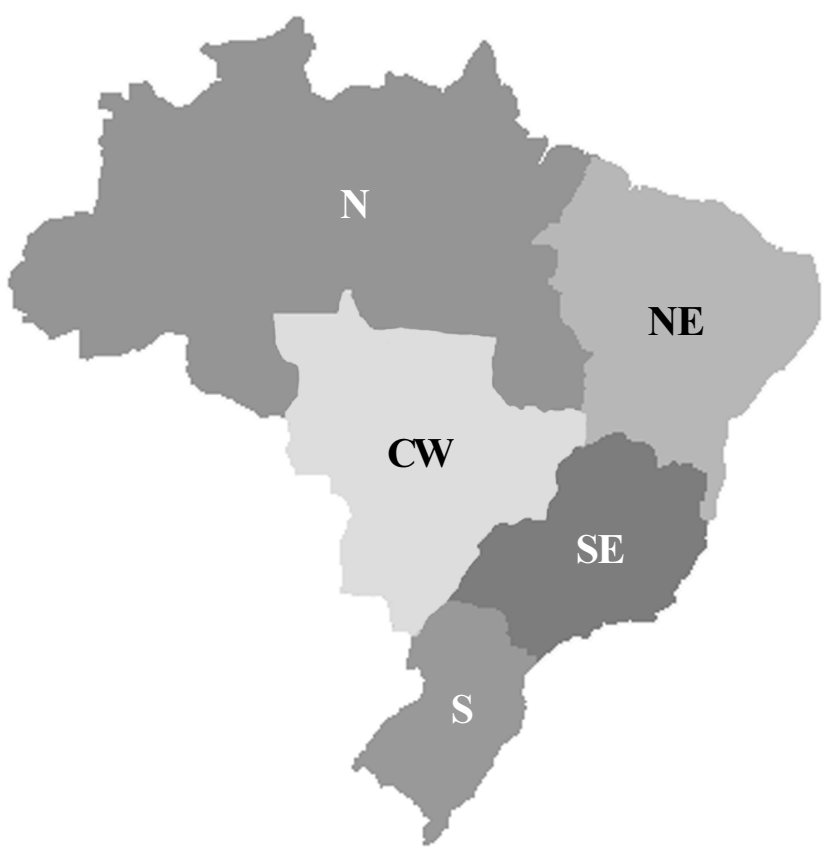

Table 2. Interpretative criteria for agents tested by E-test

\begin{tabular}{lccc}
\hline $\begin{array}{l}\text { Amtimicrobial } \\
\text { Agent }\end{array}$ & $\begin{array}{c}\text { Susceptible } \\
\mu \mathbf{g} / \mathbf{m l}\end{array}$ & $\begin{array}{c}\text { Intermediate } \\
\mu \mathbf{g} / \mathbf{m l}\end{array}$ & $\begin{array}{c}\text { Resistant } \\
\mu \mathbf{g} / \mathbf{m l}\end{array}$ \\
\hline Penicillin & $\leq 0.06$ & $0.12-1$ & $\geq 2$ \\
Amoxicillin/Clavulanate & $\leq 2$ & 4 & $\geq 8$ \\
Cefaclor & $\leq 1$ & 2 & $\geq 4$ \\
Cefotaxime & $\leq 0.5$ & 1 & $\geq 2$ \\
\hline
\end{tabular}


Table 3. Group A: S. pneumoniae susceptibility/Brazilian regions

\begin{tabular}{|c|c|c|c|c|}
\hline & $\begin{array}{c}\text { Total number } \\
\text { of isolates } \\
(\mathbf{n}=\mathbf{3 7 5})\end{array}$ & $\begin{array}{c}\text { South } \\
\text { region } \\
(n=147)\end{array}$ & $\begin{array}{c}\text { South east } \\
\text { region } \\
(n=186)\end{array}$ & $\begin{array}{l}\text { Center west } \\
\text { region } \\
(n=42)\end{array}$ \\
\hline \multicolumn{5}{|l|}{ Penicillin } \\
\hline $\mathrm{MIC}_{50}$ & $<0.016$ & $<0.016$ & $<0.016$ & $<0.016$ \\
\hline $\mathrm{MIC}_{90}$ & 0.047 & 0.032 & 0.047 & 0.064 \\
\hline$\% \mathrm{~S} / \mathrm{I} / \mathrm{R}$ & $90.1 / 9.9 / 0$ & $93.2 / 6.8 / 0$ & $89.8 / 10.2 / 0$ & $81 / 19 / 0$ \\
\hline \multicolumn{5}{|l|}{ Amox/Clav. } \\
\hline $\mathrm{MIC}_{50}$ & $<0.016$ & $<0.016$ & $<0.016$ & $<0.016$ \\
\hline $\mathrm{MIC}_{90}$ & 0.023 & 0.016 & 0.023 & 0.032 \\
\hline$\% \mathrm{~S} / \mathrm{I} / \mathrm{R}$ & $100 / 0 / 0$ & $100 / 0 / 0$ & $100 / 0 / 0$ & $100 / 0 / 0$ \\
\hline \multicolumn{5}{|l|}{ Cefaclor } \\
\hline $\mathrm{MIC}_{50}$ & 0.19 & 0.19 & 0.19 & 0.19 \\
\hline $\mathrm{MIC}_{90}$ & 0.38 & 0.38 & 0.38 & 0.38 \\
\hline$\% \mathrm{~S} / \mathrm{I} / \mathrm{R}$ & 97.6 / 0.8 / 1.6 & 96.6 / 1.4 / 2 & 97.8 / $0.6 / 1.6$ & $100 / 0 / 0$ \\
\hline \multicolumn{5}{|l|}{ Cefotaxime } \\
\hline $\mathrm{MIC}_{50}$ & $<0.016$ & $<0.016$ & $<0.016$ & $<0.016$ \\
\hline $\mathrm{MIC}_{90}$ & 0.032 & 0.023 & 0.047 & 0.047 \\
\hline$\% \mathrm{~S} / \mathrm{I} / \mathrm{R}$ & $100 / 0 / 0$ & $100 / 0 / 0$ & $100 / 0 / 0$ & $100 / 0 / 0$ \\
\hline \multicolumn{5}{|c|}{ Chloramphenicol } \\
\hline$\% \mathrm{~S} / \mathrm{I} / \mathrm{R}$ & $95.2 / 0 / 4.8$ & 93.9 / 0 / 6.1 & $95.2 / 0 / 4.8$ & $100 / 0 / 0$ \\
\hline \multicolumn{5}{|l|}{ Erythromycin } \\
\hline$\% \mathrm{~S} / \mathrm{I} / \mathrm{R}$ & $96.3 / 1 / 2.7$ & 95.2 / 1.4 / 3.4 & $97.3 / 1.1 / 1.6$ & $95.2 / 0 / 4.8$ \\
\hline \multicolumn{5}{|l|}{ Tetracycline } \\
\hline$\% \mathrm{~S} / \mathrm{I} / \mathrm{R}$ & 76.5 / 12 / 11.5 & $80.3 / 11,5 / 8.2$ & $76.3 / 12.4 / 11.3$ & $64.3 / 11.9 / 23.8$ \\
\hline \multicolumn{5}{|c|}{ Trimethoprim/Sulfamethoxazole } \\
\hline$\% \mathrm{~S} / \mathrm{I} / \mathrm{R}$ & $32 / 4 / 64$ & $34 / 4.8 / 61.2$ & 34.4 / 2.7 / 62.9 & $14.3 / 7.1 / 78.6$ \\
\hline
\end{tabular}


97.6\%; erythromycin $96.3 \%$; tetracycline $76.5 \%$; SMT-TMZ 32\% .

Results were similar among the three Brazilian regions, however, S. pneumoniae strains from the Center-West $(\mathrm{CW})$ region tended to have lower susceptibility to all antibiotics when compared to overall results (percentage of susceptibility to tetracycline $64.3 \%$ vs. $76.5 \%$, SMT-TMZ $14.3 \%$ vs. $32 \%$ ). The highest intermediate resistance rate to penicillin was also found in the $\mathrm{CW}$ region: $19.0 \%$ vs. $9.9 \%$ overall results.

Table 3 sumarizes the data from each region for each of the drugs, recorded as MICs 50 and 90.

Group B. Healthy Children Carriers ( $<5$ years old). Nasal swabs from 2,521 Brazilian children $<5$ years old were collected from participating daycare centers by the same nurse. They were forwarded to our central laboratory in São Paulo, Brazil, where they were cultured, identified, and tested against the same drug panel by the same method as in Group A.

Streptococcus pneumoniae isolates were found in 350 (13.9\%) of the 2,521 nasal swabs obtained (overall carrier rate). Among 350 different isolates, the distribution among regions was as follows: 100 from South, 130 from SW, 69 from North, and 51 from NE. High level resistance $(>2 \mu \mathrm{g} / \mathrm{ml})$ to penicillin was not found. Intermediate resistance to penicillin was observed in $32(9.2 \%)$ of the 350 strains, while the remainder (90.8\%) were susceptible. A summary of the regional distribution resistance patterns MICs 50 and MICs 90 are summarized in Table 4.

\section{Discussion}

Brazil is a country of continental proportions and it is important to define the resistance differences among regions.

S. pneumoniae isolates from patients and healthy carriers had similar rates of intermediate resistance to penicillin $(9.9 \%$ in group $\mathrm{A}$, and $9.2 \%$ in group B) when looking at overall results. No strains with high level resistance to penicillin were found in either group and the intermediate penicillin resistance levels were similar in both $(9.9 \%$ in group $\mathrm{A}$ and $9.2 \%$ in group B). Group A strains from CenterWest (CW) showed marked differences for penicillin with intermediate rate $19 \%$ when compared to $9.9 \%$ overall, and high cross resistance rates to tetracycline $(23.8 \%)$ and trimethoprim/sulfamethoxazole (78.6\%). This group represented diverse patients with very littlle clinical information other than specimen and patient's age, so it was difficult to draw any epidemiological conclusions regarding resistance differences in the $\mathrm{CW}$. This should be looked at more carefully in further studies. Laboratories from the North region enrolled to participate encountered difficulties and did not submit any strains.

Group B penicillin strains with intermediate resistance rates from SE (13.8\%), NE (9.8\%) and South $(8.0 \%)$ were quite similar, but the North region showed a markedly low percentage $1.4 \%$ of intermediate resistance when compared to other surveillance studies in Brazil [6]. Erythromycin overall resistance was $4.6 \%$, but the North region had a resistance rate of $8.7 \%$. Information regarding antibiotic comsumption remains a challenge to record due to a lack of consistent information, but efforts should be made to clarify those major resistance differences.

The accelerated increase in penicillin resistance observed over the last 10 years in other countries, is also a reality in Brazil even though resistance trends have been slower. In the LASER Group study [7], where $1,100 \mathrm{~S}$. pneumoniae isolates were tested, Brazil showed the highest prevalence of penicillin susceptible organisms (87.1\%), followed by Argentina (80.9\%), Venezuela (78.1\%), Panama (77.0\%) and Chile (68.7\%).

Most respiratory tract infections are treated on an empirical basis, so it is necessary to keep monitoring patterns of S. pneumoniae resistance, especially in local regions and communities, to better guide antibiotic therapy.

The prevalence of resistance to non-b-lactam agents also increased significantly in penicillin susceptible and penicillin intermediate strains [7,8]. It is important to highlight that the breakpoints for amoxycillin/clavulanate, erythromycin, tetracycline, TMP-SXZ, and cefaclor are 
Table 4. Group B - carriers: S. pneumoniae susceptibility patterns/Brazilian regions

\begin{tabular}{|c|c|c|c|c|c|}
\hline & $\begin{array}{c}\text { Total number of } \\
\text { isolates } \\
(n=350)\end{array}$ & $\begin{array}{l}\text { South } \\
\text { region } \\
(\mathbf{n}=\mathbf{1 0 0})\end{array}$ & $\begin{array}{l}\text { South east } \\
\text { region } \\
(n=130)\end{array}$ & $\begin{array}{l}\text { North } \\
\text { region } \\
(n=69)\end{array}$ & $\begin{array}{l}\text { North east } \\
\text { region } \\
(n=51)\end{array}$ \\
\hline \multicolumn{6}{|l|}{ Penicillin } \\
\hline $\mathrm{MIC}_{50}$ & $<0.016$ & $<0.016$ & $<0.016$ & - & $<0.016$ \\
\hline $\mathrm{MIC}_{90}^{30}$ & 0.047 & 0.047 & 0.064 & $<0,016$ & 0.047 \\
\hline$\% \mathrm{~S} / \mathrm{I} / \mathrm{R}$ & $90.8 / 9.2 / 0$ & $92.0 / 8.0 / 0$ & $86.2 / 13.8 / 0$ & $98.6 / 1.4 / 0$ & $90.2 / 9.8 / 0$ \\
\hline \multicolumn{6}{|l|}{ Amox./Clav. } \\
\hline $\mathrm{MIC}_{50}$ & $<0.016$ & $<0.016$ & $<0.016$ & - & $<0.016$ \\
\hline $\mathrm{MIC}_{90}$ & 0.016 & 0.016 & 0.032 & $<0,016$ & 0.016 \\
\hline$\% \mathrm{~S} / \mathrm{I} / \mathrm{R}$ & $100 / 0 / 0$ & $100 / 0 / 0$ & $100 / 0 / 0$ & $100 / 0 / 0$ & $100 / 0 / 0$ \\
\hline \multicolumn{6}{|l|}{ Cefaclor } \\
\hline $\mathrm{MIC}_{50}$ & 0.19 & 0.19 & 0.19 & 0.125 & 0.19 \\
\hline $\mathrm{MIC}_{90}$ & 0.38 & 0.38 & 0.5 & 0.25 & 0.5 \\
\hline$\% \mathrm{~S} / \mathrm{I} / \mathrm{R}$ & $97.4 / 0.3 / 2.3$ & $96.0 / 0 / 4$ & $96.1 / 0.8 / 3.1$ & $100 / 0 / 0$ & $100 / 0 / 0$ \\
\hline \multicolumn{6}{|l|}{ Cefotaxime } \\
\hline $\mathrm{MIC}_{50}$ & $<0.016$ & $<0.016$ & 0.016 & - & 0.016 \\
\hline $\mathrm{MIC}_{90}$ & 0.047 & 0.047 & 0.047 & 0.016 & 0.047 \\
\hline$\% \mathrm{~S} / \mathrm{I} / \mathrm{R}$ & $100 / 0 / 0$ & $100 / 0 / 0$ & $100 / 0 / 0$ & $100 / 0 / 0$ & $100 / 0 / 0$ \\
\hline \multicolumn{6}{|c|}{ Chloramphenicol } \\
\hline$\% \mathrm{~S} / \mathrm{I} / \mathrm{R}$ & $95.7 / 0 / 4.3$ & $94 / 0 / 6$ & $94.6 / 0 / 5.4$ & $100 / 0 / 0$ & $96.1 / 0 / 3.9$ \\
\hline \multicolumn{6}{|l|}{ Erythromycin } \\
\hline$\% \mathrm{~S} / \mathrm{I} / \mathrm{R}$ & $94.3 / 1.1 / 4.6$ & $98 / 1 / 1$ & $93.1 / 7$ / 6.2 & $91.3 / 0 / 8.7$ & $94.1 / 3.9 / 2$ \\
\hline \multicolumn{6}{|l|}{ Tetracyclin } \\
\hline$\% \mathrm{~S} / \mathrm{I} / \mathrm{R}$ & $78 / 9.4 / 12.6$ & $83 / 13 / 4$ & $74.6 / 7.7$ / 17.7 & 78.3 / 10.4 / 11.6 & $76.5 / 5.9 / 17.6$ \\
\hline \multirow{2}{*}{\multicolumn{6}{|c|}{$\begin{array}{l}\text { Trimethoprim/ } \\
\text { Sulfametoxazol }\end{array}$}} \\
\hline & & & & & \\
\hline$\% \mathrm{~S} / \mathrm{I} / \mathrm{R}$ & $16 / 5.1 / 78.9$ & 14 / 4 / 82 & $18.5 / 5.3 / 76.2$ & 17.4 / 2.9 / 79.7 & $11.8 / 9.8 / 78.4$ \\
\hline
\end{tabular}


based on oral use of these agents, while the breakpoints for penicillin and ceftaxime are primarily based on standard intravenous dose administration for meningeal infections. The results obtained for amoxycillin/ clavulanate are identical to those obtained with amoxycillin against $S$. pneumoniae as this organism does not produce beta-lactamase.

Resistance to macrolides has increased worldwide however, in this study, we did not find such high resistance rates (in either group) as can be seen in some Latin American countries like Mexico [7].

From a clinical point of view, it is important that treatment of pneumococcal infections in different clinical sites should also be considered when we analyze surveillance results. In vitro data should always be considered with regards to the site of the infection as well as to demographic data in order to choose which drug is the most appropriate for therapy. Cefotaxime or ceftriaxone plus vancomycin have been recommended for the treatment of meningitis caused by penicillin nonsusceptible strains [9] Imipenem and meropenem have been found to be effective in reports of meningitis due to penicillin resistant strains $[10,11]$. In adults with pneumococal bacteremia, high dose iv penicillin $G$ $(150,000$ to $250,000 \mathrm{U} / \mathrm{kg}$ per day) has been recommended for penicillin intermediate strains [12].

Resistance in Streptococcus pneumoniae creates some problems for the physician when empiric treatment has to be initiated. It is widely accepted that there is inadequate awareness regarding treatment of penicillin non-susceptible pneumococcal infections due to misinterpretations of the current breakpoints to different clinical situations. The Drug-Resistant Streptococcus pneumoniae Therapeutic Working Group (DRSTWG) of the Centers for Disease Control (CDC) has recently made recommendations for treatment of acute otitis media (AOM) in which amoxicillin is indicated as the first choice for uncomplicated cases of AOM. For high risk patients (e.g. patients with recent antimicrobial exposure, $<2$ years of age, and/or in day care setting), high doses of amoxicillin or amoxicillin-clavulanate (up to $70-90 \mathrm{mg} / \mathrm{kg} /$ day of amoxicillin) are currently recommended. For treatment failures with empiric amoxicillin, an antibiotic that is effective for treatment of $\beta$-lactamase producing organisms (H. influenzae), and is also active against DRSP, should be prescribed. These would include amoxicillin-clavulanate (especially if given athigher doses of the amoxicillin component), cefuroxime axetil, and intramuscular ceftriaxone. Tympanocentesis may be performed in refractory cases of otitis media in order to obtain evidence of the causative pathogen as well as to determine the susceptibility of the organism give the clinician may a microbiological support when deciding on the appropriate therapy in each case [13] .

Treatment of respiratory infections is a great challenge because microbiological cultures and sensitivities are usually not routinely requested and lack of appropriate methods is another important issue in Brazil. Surveillance studies [6,14] are an important epidemiological tool for clinicians to help decide which is the most appropriate empiric treatment. Local microbiological data supports appropriate use of antimicrobial agents. The new breakpoints for oral drugs show better correlation with clinical outcomes, as demonstrated by Craig and others [13,15].

Local surveillance protocols should be run on a continuous basis and standardized microbiological methods should always be considered. Information about associated resistance, as well as differences in prevalence associated with region, specimen source, and patient age is extremely important. Efforts toward a policy on appropriate antimicrobial prescribing should be an important multidisciplinary target to ensure that only the the best agents most appropriate drugs are used.

\section{Acknowledgements}

We thank Cassia Zoccoli, Jorge Sampaio Caio M. F. Mendes, Adília Segura, Elza Mamizuka, Tereza Bandeira, Evangelina Araújo, Afonso Barth, Bruno Bertshinger, and Cassio Murilo Villefort, for the strains they provided from their clinical laboratories in Brazil, and to Fernando Coimbra, Jose Luiz Tavares, Katia Maria C Pires, Paulo Ribeiro Jr. from SmithKline Beecham Brazil. This study was funded, in part, by a research grant from SmithKline Beecham Pharmaceuticals, Inc. 


\section{References}

1. Appelbaum P.C. Antimicrobial Resistance in Streptococcus pneumoniae:An Overview. Clin Infect Dis 1992;15:77-83.

2. Di Fabio J.L., Homma A., DeQuadros C. Pan American Health Organization Epidemiological Surveillance Network for Streptococcus pneumoniae. Microb Drug Resistance 1997: 133.

3. National Committee for Clinical Laboratory Standards (NCCLS). Performance Standards for Antimicrobial Susceptibility Testing. NCCLS documents M7-A5, M2A7, table M100S10. Fifth Edition, 2000.

4. Simpson I., Alexander Group. The Alexander Project An International multi-centre survey of the antimicrobial susceptibilities of Streptococcus pneumoniae, Haemophilus influenzae, Moraxella catarrhalis and Staphylococcus aureus. 1996-2000.

5. Murray P.R., Baron E.J., Pfaller M.A., et al. Manual of Clinical Microbiology. 7th ed. American Society for Microbiology, 1999.

6. Brandileone M.C., Simonsen D.V., Casagrande S., et al. Prevalence of serotypes and antimicrobial resistance of Streptococcus pneumoniae strains isolated from Brazilian children with invasive infections. Microb Drug Resistance 1997;3:141-6.

7. Jacobs M.J., Appelbaum P. LASER Study Group. Susceptibility of $1100 \mathrm{~S}$. pneumoniae strains isolated in 1997 from seven Latin American And Caribbean countries. International Jounal of Antimicrobial Agents 2000; 17-24.

8. Critchley I., Thormsberry C., et al. Antimicrobial susceptibility of S. pneumoniae, Haemophilus influenzae and Moraxella catharrallis collected from five centers in Brazil,1997-98.Clin Microbio Infect 2000;6:178-84.

9. McCraken G.H., Nelson J.D., Kaplan S.L., et al. Consensus report: antimicrobial therapy for bacterial meningitis in infants and children. Pediatr Infect Dis J 1987;6:501-5.

10. McCracken G.H., Sakata Y. Antimicrobial therapy of experimental meningitis caused by Streptococcus pneumoniae with different susceptibilities to penicillin. Antimicrob Agents Chemother 1985; 27:141-5.

11. Lopes E., Meropenem Study Group. Meropenem versus cefotaxime or ceftriaxone for bacterial meningitis [abstract 638]. In: Progam and Abstracts of the 34th Intercience Conference on Antimicrobial Agents and Chemotherapy. Washington, DC: American Society for Microbiology, 1993:236.

12. Pallares R., Gudiol F., Linares J., et al. Risk factors and response to antibiotic therapy in adults with bacteremic pneumoniae caused by penicillin-resistant pneumococci. N Engl J Med 1987;317:18-22.
13. Doewll S.F., Bluter J.C., Giebink G.S., et al. Acute Otitis Media: Management and surveillance in an era of pneumcoccal resistance: a report from the drug-resistant Streptococcus pneumoniae therapeutic working group (DRSPTWG). Pediatr Infect Dis J 1999;18:1-9.

14. Doern G.V., Pfaller M.A., Kugler K., et al. Prevalence of antimicrobial Resistance among Respiratory Tract Isolates of Streptococcus pneumoniae in Notrh America: 1997 Results from the Sentry Antimicrobial Surveillance Program. Clin Infect Dis 1998;27:764-70.

15. Craig W.A., Andes D. Pharmacokinetics and Pharmacodynamics of antibiotics in Otitis Media. Pediatr Infect Dis J 1996; 15:255-9. 